\title{
Discussions on Novel Inverters Constructed by Minimum Components
}

\section{- Pursuit for simple power conditioner -}

\author{
Keiju Matsui ${ }^{a^{*}}$, Eiji Oishi $^{\mathrm{a}}$, Masayoshi Umeno ${ }^{\text {a }}$, \\ Mikio Yasubayashi ${ }^{\mathrm{b}}$, Hideo Uchida $^{\mathrm{b}}$, Masaru Hasegawa ${ }^{\mathrm{b}}$ \\ ${ }^{a}$ Minna-denryoku,Inc. Setagaya Monozukuri Gakko, 210 Setagaya 154-0001, Japan \\ ${ }^{\mathrm{b}}$ Chubu University Kasugai 487-8501, Japan
}

\begin{abstract}
Photovoltaic power generations (PVG) have been accepted and spread widely. Various power conditioning systems used in them have been also studied by many researchers. In general utilization, the actual application of such solar panels are almost installed on top of the roof of isolated house. Some residents living in the apartment house, however, have fairly desires to contribute for energy saving due to natural energy generation. The generating power in such case is fairly reduced, so the system construction should balance the reduced power. Thus, it is necessary to improve the construction toward simple one. In this paper, in order to give a reply, simple and concise power conditioners, especially novel inverter is proposed. Considering fairly reduced generating power and narrow space of installation, the system constructions should be compact. The circuits which gratifies their operating characteristic is presented and analytically discussed about circuit construction as a novel converter.
\end{abstract}

Keywords: Power Conditioner, PCS, Solar cell, Minimum construction, Buck-boost inverter, Boost inverter, Half bridge, photovoltaic power generation

\section{Introduction}

It is an important assignment for humanity of the world to conquer the problems against environmental conservation with preserving the developments of economy, science and technology. The usage of the renewable energies is expected to be able to mitigate such problem, which is an important strategies [1,2]. Among them, photovoltaic power generation have various advantages such as usage of inexhaustible and unpolluted sunlight, which is advantageous for maintenances, easily construction from small power to large one, and can be installed in various location including city center. In such a way, this power generation system is a promising one with bright future.

As a utilization form, utility interactive power generation system has been accepted and widely spread. In such system, it is necessary to install the chopper, where the lower voltage of solar cell should be boosted to suitable voltage, and inverter where the dc power of boosted voltage should be converted to ac power in the power system $[3,4]$.

The power conditioners (PCS) including inverter have been presented in various systems so far $[5,6]$. However, it is necessary to reduce the cost even more. It is said that the system is approaching to an ideal ones with respect to efficiency and construction strategy, but that cost would prevent wide spread if there is no public financial support. In such discussions, there are many subjects to be solved to utilize the PV power in utility interactive power generation. Even more, various safeguard equipment required according to regulations make the cost increase. Thus, it is required to obtain even lower cost PCS. In an extremely reduced power PCS as discussed in this paper, a way of handling would be different compared to typical power range. In such case of reduced generating power, quantities of reversed power to the power system would be small, where another mitigated regulation or deregulation might be approved. Thus, in such PV power generation systems, there are so many subjects to be resolved [7].

Under such circumstances accepted by electrical utility industry as recognitions and assessments for renewable energy, spread of application is strongly sustained by financial supports of public organizations, and a lot of consumers are hoping to install such PV power generation system. For ordinary homes, installation of solar panel is restricted to house having roofs. However, a lot of residents living in the apartment house also wish to install the PV panel under considering resource conservation with such public financial support. In the present situation, however, there is no scheme to perform these requirements. The authors have been studied in a series of the small power PV system [8,9]. In this paper, some simple PCS systems, especially the components like inverters and chopper are integrated toward simple construction, which are presented and discussed. 


\section{Conception and its Object of Unified BC-INV \\ Summary of circuit configuration [10]}

Under a few hundred watts of small photovoltaic power generation system (PGS), it is important to pursuit for simple circuit configuration in proportion to small power. In order to realize this situation, the unified chopper and inverter circuit configuration came to an idea to the authors. In this strategy, one of the conventional inverter switches was also used for the switch of boosting chopper, which brings a simple and low cost configuration.

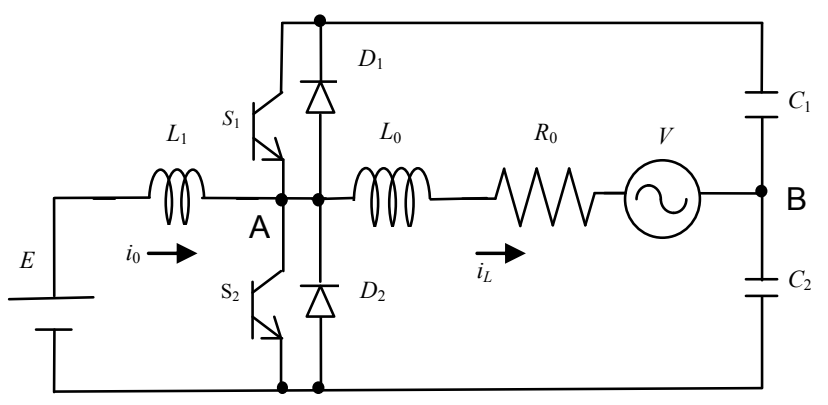

Fig.1. Previously presented PCS constructed by half bridge[10].

The remarkable characteristic is to obtain the boosted voltage inverter function, by means of merely changing the connecting point of the input power supply from the dc link terminal which is the midpoint of inverter leg. By such a way, simple BC-INV can be obtained in a very simple manner. By means of this, the dc link voltage becomes double and an unique sinusoidal inverter can be obtained in half bridge constructed by the minimum circuit components.

However, the applied voltage across the chalk inductor $\mathrm{L}_{1}$ becomes $v_{L 1}=v_{A B}$, where the commercial frequency is generated in the chalk inductor. Consequently, the size of $\mathrm{L}_{1}$ would become much large. Because of such reasons, for practical applications, there is a problem, so in practice, it could be applied for current source inverter and the like, so the application is limited to restricted fields.

\section{Further Development to Novel Inverter having Minimum Components}

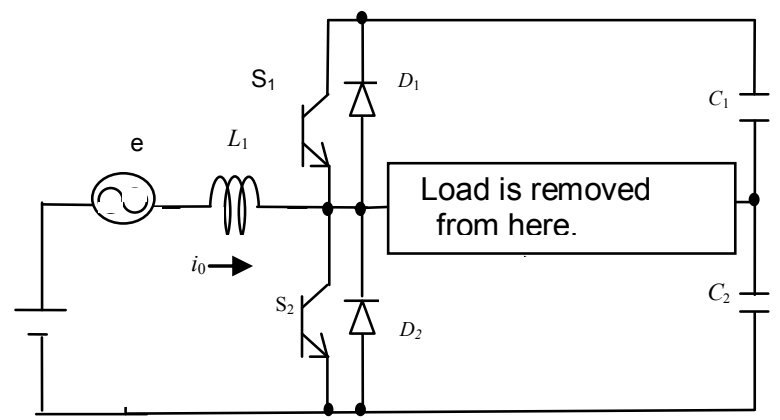

Fig.2. Simple Construction in Minimum Components for case 1 .

\subsection{First version of novel inverter in minimum components}

Fig.2 shows the first version of novel inverter constructed by minimum component. From the fact that the applied voltage across the inductor $L_{1}$ in Fig. 1 is the commercial frequency, the idea is arisen if ac load might be installed at this corresponding place.

The relationship of input and output voltage can be obtained as follows; The modulation method is given by "Natural Sampling (NS) method". Let the modulation index be $\alpha$, so the ac load voltage becomes

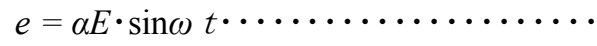

The following relation can be obtained.

(a) for $e>0$

- $\mathrm{S}_{2}$ is turned-on,

$$
E=v_{L 1}+e \quad \cdots \cdots \ldots \ldots \ldots \ldots
$$

- at $\mathrm{S}_{2}$ is turned-off,

$$
E+v_{L 2}=e+v_{C} \quad \cdots \cdots \cdots \cdots \cdots
$$

where $v_{L 1}$ and $v_{L 2}$ are considered for their polarities of applied voltage.

In the NS method which has positive and negative polarity triangular waves, each pulse width of the output voltage given by positive and negative waves is varied in $(1+\alpha)$ or $(1-\alpha)$, where one cycle is given by normalized $T$ $=2$. By using "Inductor Equal Area Rule" for positive and negative voltage waveforms,

$$
(E-e)(1+\alpha)=\left(e-E+v_{C}\right)(1-\alpha) \cdots
$$

From this result,

$$
v_{C}=(2 E-2 e) /(1-\alpha)
$$

The value of $e=\alpha E$, where $e$ is constant during corresponding period, is substituted, then

$$
v_{C}=2 E
$$

The result can be obtained clearly.

(b) for e $<0$

- $\mathrm{S}_{1}$ is turned-on.

$$
v_{C}=v_{L 1}+e+E
$$

- $\mathrm{S}_{1}$ is turned-off.

$$
v_{L 2}=e+E
$$

where $v_{L 1}$ and $v_{L 2}$ are applied voltage across inductor considering their polarities.

In similar as above mentioned, 


$$
\left(v_{C}-E-e\right)(1+\alpha)=(e+E)(1-\alpha) \cdots \cdots
$$

Thus,

$$
v_{C}=(2 E+2 e) /(1+\alpha)
$$

Substituting $e=\alpha E$ into (10),

$$
v_{C}=2 E
$$

In this equation, the relationship in voltage ratio between input and output is two times. During period of (b) $e<0$, however, there is a power regenerative period, when power is returned from $\mathrm{V}_{\mathrm{C}}$ to $\mathrm{E}$. In order to improve this phenomenon, the current flowing route is altered.

\subsection{Second version of novel inverter in minimum component}

Fig.3 shows second version of novel inverter in minimum component obtained by modifying Fig.2.

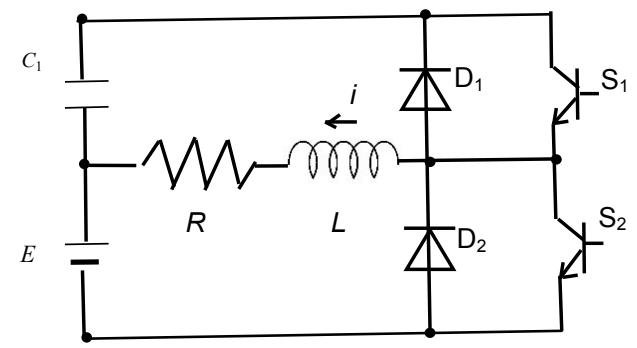

Fig.3. Simple Construction in Minimum Component for case 2.

In the first version of Fig.2, the voltage of auxiliary double capacitors providing the negative load current, is given by $V_{C}=2 E$, which brings the regenerative power flow. In order to avoid this phenomenon, a modified circuit configuration is provided as in Fig. 3.

Instead of using the output capacitor $C_{1}$ and $C_{2}$ like in Fig.2, the capacitor $C_{1}$ in Fig. 3 is used as auxiliary one which can be easily derived from Fig.2. The operation can be performed as follows;

The modulation method is used in similar to the first version of single triangular wave with positive and negative polarities, that is NS method. The following relationship can be obtained.

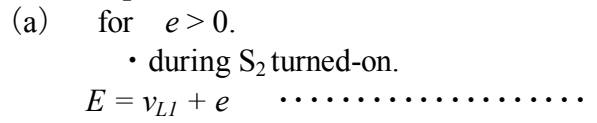

- during $\mathrm{S}_{2}$ turned-off.

$v_{L 2}=e+v_{C 2} \quad \ldots \ldots \ldots \ldots \ldots \ldots$

where $v_{L 1}$ and $v_{L 2}$ are considered for their polarities of applied voltage.

In the NS method, each pulse width of the output voltage is varied in $(1+\alpha)$ and $(1-\alpha)$. By using "Inductor Equal Area Rule".

$$
(E-e)(1+\alpha)=\left(E+v_{C 2}\right)(1-\alpha) \cdots \cdots \cdots \cdot(14)
$$

From this result, following equation can be obtained.

$$
\begin{aligned}
& v_{C 2}=(E-e) /(1-\alpha) \quad \cdots \cdots \cdots \cdots \cdots \cdots \cdots \\
& v_{C}=E \quad \cdots \cdots \cdots \cdots \cdots \cdots \cdots \cdots
\end{aligned}
$$

(c) for $e<0$.

- during $\mathrm{S}_{1}$ turned-on

$$
v_{C}=v_{L I}+e
$$

$\cdot$ during $\mathrm{S}_{1}$ turned-off

$$
v_{L 2}=e+E
$$

In a similar way as above mentioned,

Thus,

$$
\left(v_{C}-e\right)(1+\alpha)=(e+E)(1-\alpha)
$$

$$
v_{C}=(E-\alpha E+2 e) /(1+\alpha)
$$

In substituting $e=\alpha E$ into this equation,

$$
v_{C}=E
$$

The relationship can be obtained clearly that the voltage between input and output becomes equal. The disadvantageous characteristic of output voltage double can be revised, where there is no regenerative operation. Consequently, that capacity of output capacitor can be reduced.

\subsection{Power delivery flowchart}

Fig. 4 shows the power flowchart for the operation of circuit shown in Fig.3. It can be seen that is more effective strategy in comparison both circuits. In the figure, the power flows from dc power supply $\mathrm{E}$ through inductor $\mathrm{L}$ and load $\mathrm{R}$ to auxiliary capacitor C. $1-\mathrm{d}$ or $1+\mathrm{d}$ represents normalized conduction period.

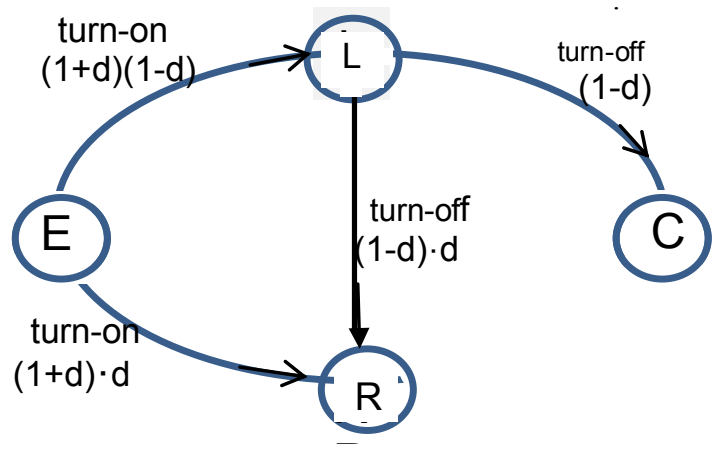

Fig.4. Power Flowchart of Proposed Inverter.: input supply E to capacitor $\mathrm{C}$.

(a) Explanation about power flowchart

Let one switching cycle be $T=2$ in normalization. During switch $\mathrm{S}_{2}$ turned-on, that is $(1+d)$, the voltage, $\left(E-v_{R}\right)=E(1-d)$ is applied across the $\mathrm{L}$ and provide the magnetic energy, that is, $E(1+d) \times(1-d)$.

During switch $\mathrm{S}_{2}$ turning-off, that is $(1-d), \mathrm{R}$ is supplied due to discharged L and the auxiliary capacitor is supplied as follows;

$$
(1-d) \times E(1+d)=(1-d) \times\left(v_{C}+v_{R}\right) \cdots \cdots
$$

From these equations, the relationship of $v_{C}=E$ can be obtained, where the auxiliary capacitor voltage becomes equal to the dc supply voltage.

(b) Explanation of power transmission in power sharing by inductor

The stored energy in $\mathrm{L}$ at turned-on is delivered to the load $\mathrm{R}$, and then to $\mathrm{C}$ at turned-off. The stored energy $W_{L}$ in $\mathrm{L}$ is also delivered to $\mathrm{C}$ which is returned to the load again as $W_{R}$. Because of this reason, it is required to be given by the relationship of $W_{L}$ $\geq W_{R}$. These equations can be obtained as follows; The delivered 
where equation is normalized by $E$ and the last term $(\times I \Delta T)$ is abbreviated from this equation.

This value is applied to the above mentioned equation. The pulsewidth is varied according to the signal of $\alpha \sin \theta$ At $\theta=0$, the pulsewidth $\Delta T_{+}(\theta)$ becomes $\Delta T / 2$ as shown like,

$$
\Delta T_{+}(\theta)=1 / 2+(1 / 2) \alpha \sin \theta
$$

During turned-on period, the stored energy in L is

$$
\begin{aligned}
W_{L} & =\int \Delta W_{L} \cdot \Delta T_{+}(\theta) \mathrm{d} \theta \\
& =(\pi / 2)\left(1-\alpha^{2} / 2\right) \cdots
\end{aligned}
$$

In the next step, the transmission power to $\mathrm{C}$ is to be solved. The pulsewidth during turned-off is

$$
\Delta T_{-}(\theta)=1 / 2-(1 / 2) \alpha \sin \theta \cdots \cdots \cdots \cdots
$$

By using the previously mentioned relationship of $v_{C}=E$, this equation is integrated and solved the stored energy $W_{C}$ in C such as,

$$
\begin{aligned}
\mathrm{W}_{\mathrm{C}} & =\int \Delta T_{-}(\theta) \mathrm{d} \theta \\
& =(\pi / 2)(1-2 \alpha / \pi) \cdots \ldots \ldots \ldots \ldots \ldots
\end{aligned}
$$

In reference to (22), the delivered power can be obtained as the mentioned $\Delta W_{R}$

$$
\begin{aligned}
W_{R} & =\int \Delta W_{R} \mathrm{~d} \theta \\
& =2 \alpha \cdots \ldots \ldots \ldots \ldots \ldots \ldots \ldots \ldots
\end{aligned}
$$

From relation of $W_{C} \geqq W_{R}$

$$
\begin{aligned}
& (\pi / 2)(1-2 \alpha / \pi) \geqq 2 \alpha \\
& \alpha \leqq 0.524 \cdots \ldots .
\end{aligned}
$$

It can be seen from this equation that in the proposed power transmission mechanism through $\mathrm{L}$ and $\mathrm{C}$, it is required to be given by the reduced modulation index

\section{Another Development towards High Frequency Inverter}

\subsection{Power flowchart}

Fig.5 shows another circuit configuration having paralleled load in contrast series load in Fig.3. In comparison to the conventional high frequency inverter, where in half bridge circuit using boosted chopper, the obtained high frequency is rectified as dc-dc converter [12], the proposed configuration seems to be the same as the number of the circuit component

Fundamental construction can be obtained in Fig.1. With regard to the efficiency, however, the power conversion process of the proposed one is simple and the number of conversion stage is reduced, so the proposed circuit is prominent in comparison. Those validities are confirmed as follows;

In the figure, in contrast to above mentioned series load circuit, in parallel load, the sinusoidal wave cannot be obtained. Regarding rectangular wave in high frequency, it is more effective. When the switch $\mathrm{S}_{2}$ is turned-on at the beginning, the power supply provides the load resistor $\mathrm{R}$ and inductor $\mathrm{L}$ in parallel connection. Secondly, when $\mathrm{S}_{2}$ is turned-off and $\mathrm{S}_{1}$ is turned-on, the stored energy in $\mathrm{L}$ is provided to $\mathrm{R}$ and at the same time the excess energy is delivered to the auxiliary capacitor. During last half period, as the supplied power from L is reduced gradually, a deficiency for supplied power from $\mathrm{L}$ is provided from auxiliary capacitor $\mathrm{C}$. Looking at the whole period, it can be seen that the contribution for power transmission through inductor $\mathrm{L}$ is predominant. On the other hand, the power transmission through $\mathrm{C}$ is a role of auxiliary power transmission. The percentage of power transmission from $\mathrm{L}$ to $\mathrm{R}$ is about $30 \%$ at usual specification, while one from $\mathrm{L}$ to $\mathrm{R}$ through $\mathrm{C}$ is $20 \%$. For your information, the one of the direct transmission $\mathrm{E}$ to $\mathrm{R}$ is $50 \%$. It is interesting that the auxiliary capacitor $\mathrm{C}$ plays a role of like filter.

In Fig.6, the power line is plotted by heavy solid line, where transmitted power is fairly large, while thin line represents small power transmission. Looking at the whole period, it can be seen that the contribution for power transmission consists of direct transmission from $\mathrm{E}$ to $\mathrm{R}$. In addition, the route through inductor $\mathrm{L}$ is also predominant. It is remarkable strategy that the power transmission is constructed by the direct transmission. Furthermore, as another route, one is via-point of $\mathrm{L}$ and the other is via-points of $\mathrm{L}$ and $\mathrm{C}$.

Fig.7 shows the power flow chart for the conventional high frequency inverter reported as BHB-Boost Half Bridge [12], in which the circuit mechanism is compared and discussed with our strategy. The input power supply voltage is boosted in the half bridge inverter. After this operation, the high frequency ac power is obtained. After the energy of input power supply $\mathrm{E}$ is stored into $\mathrm{L}$, the capacitor $\mathrm{C}$ is charged from power from $\mathrm{L}$ at switch turned-off. By means of this capacitor charge, the load $\mathrm{R}$ is supplied. The figure shows the flowchart of such operating mechanism, where there are three step power transmissions. On the other hand, for the proposed high frequency conversion, main transmission is performed by direct conversion from $\mathrm{E}$ to load, the other is a simple transmission way via single $L$ or C. The number of conversion stage is much reduced. Consequently, the transmission efficiency can be expected as much improvement.

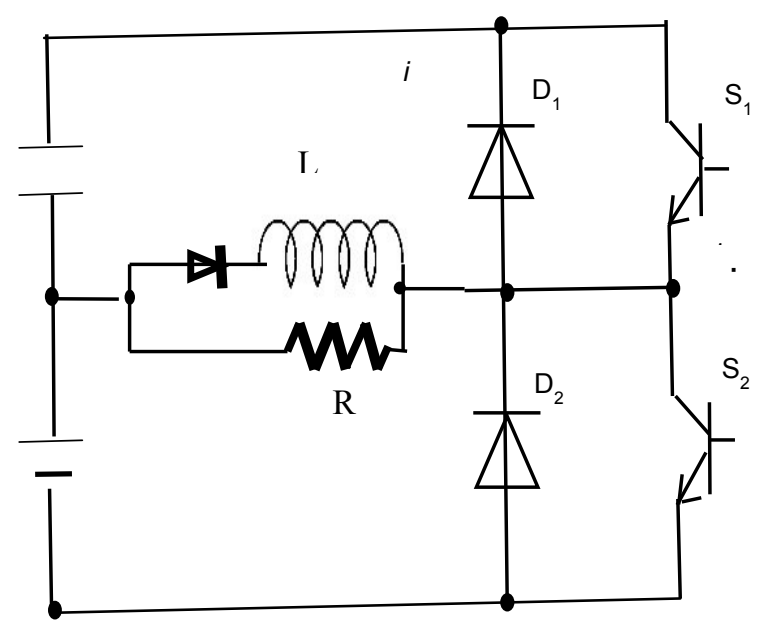

Fig.5. Proposed Circuit with Parallel Loads. 


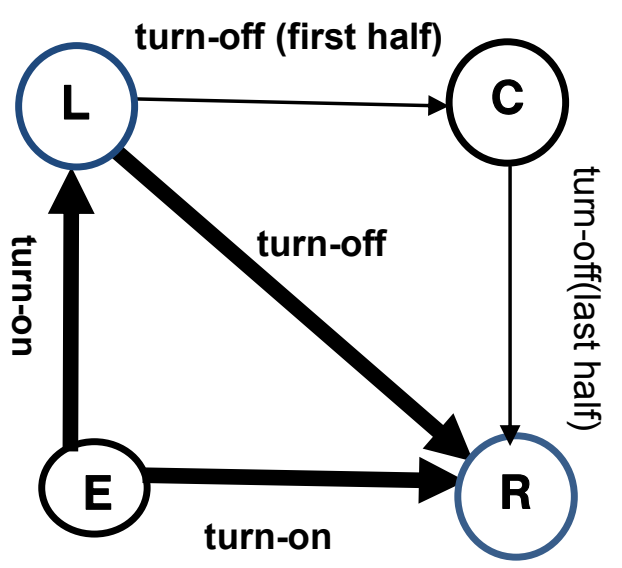

Fig.6. Power Flow for with Parallel Loads.

\subsection{Development towards buck inverter}

For further development with minimum circuit component, another method can be derived as shown in Fig.8. By means of alternating switching of $S_{1}$ and $S_{2}$ the power is delivered to the load $\mathrm{R}$. If the inductor $\mathrm{L}$ is connected in series to $\mathrm{R}$, the sinusoidal wave can be obtained. In this figure, however, the rectangular wave is kept in mind. Unbalanced charge of $\mathrm{C}$ is to be concerned, but it can be seen that balanced charge characteristic is realized by following discussion. In steady state condition,

when $\mathrm{S}_{1}$ is turned on

$$
E-v c(0)=R i^{+}
$$

when $\mathrm{S}_{1}$ is turned off

$$
v c(0)=R i^{-}
$$

where $v c(0)$ is operating voltage of $\mathrm{C} 、 i^{+}$; is charging current, and $i^{-}$is discharging current.

- In steady state condition, as $i^{-}=i^{-}$

$$
v c(0)=E / 2 \quad \mathrm{C} \text { is charged by half of supply voltage. }
$$

- In transient condition,

$$
\text { when } \mathrm{S}_{1} \text { is turned on, }
$$

$E-v c(t)=R C \cdot \mathrm{d} v c(t) / \mathrm{d} t$,

when $\mathrm{S}_{1}$ is turned off

$$
v c(t)=-R C \cdot \mathrm{d} v c(t) / \mathrm{d} t
$$

When the gradient of $\mathrm{C}, \mathrm{d} v c(t) / \mathrm{d} t$ becomes equal, relationship of $E=v c(t) / 2$ establishes and $\quad \mathrm{C}$ is charged by $\mathrm{E} / 2$.

\subsection{Flowchart of buck inverter}

In Fig.9, at $\mathrm{S}_{1}$ turned-on and $\mathrm{S}_{2}$ turned-off, the load $\mathrm{R}$ and $\mathrm{C}$ are supplied simultaneously. When $\mathrm{S}_{1}$ is turned off and $\mathrm{S}_{2}$ turned on, the $\mathrm{R}$ is supplied from power of $\mathrm{C}$. The half power from $E$ is directly supplied to load $R$ which contributes to efficiency improvement that is rather advantageous.

Fig. 10 shows the flowchart of conventional half bridge inverter for comparison. For half bridge, $\mathrm{C}$ is connected in series with supplied power, image of sequential power transmission may be occurred. But actually, the circuit is changed by $\mathrm{C}_{1}$ and $\mathrm{C}_{2}$, and power is delivered by two steps.

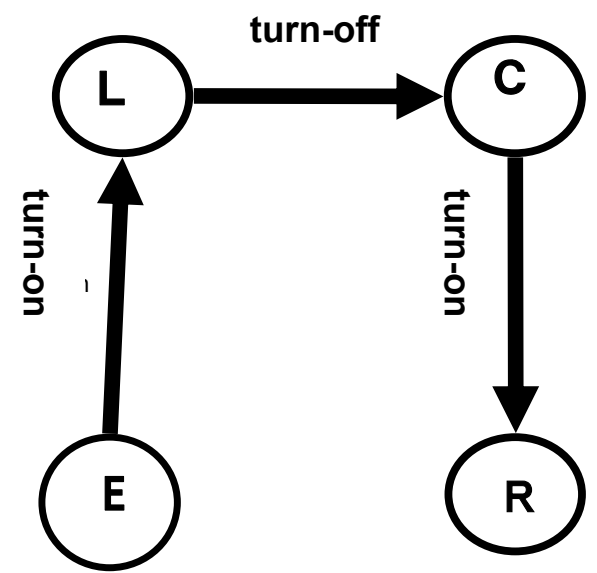

Fig.7. Conventional Half Bridge Power Transmission.

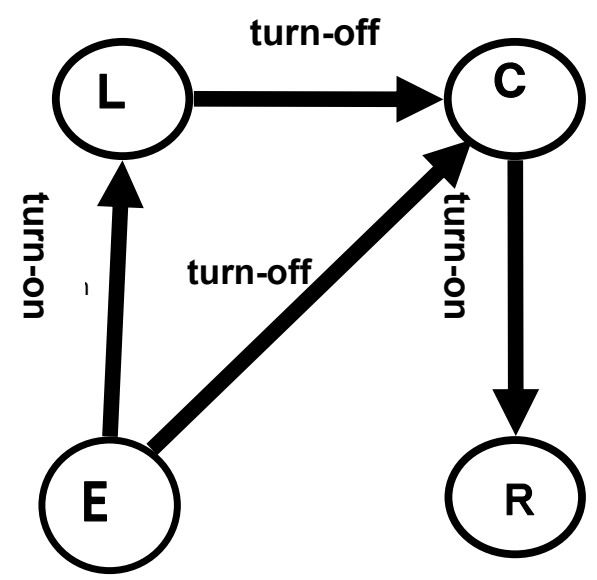

Fig.7. Boost Half Bridge Power Transmission ${ }^{(12)}$.

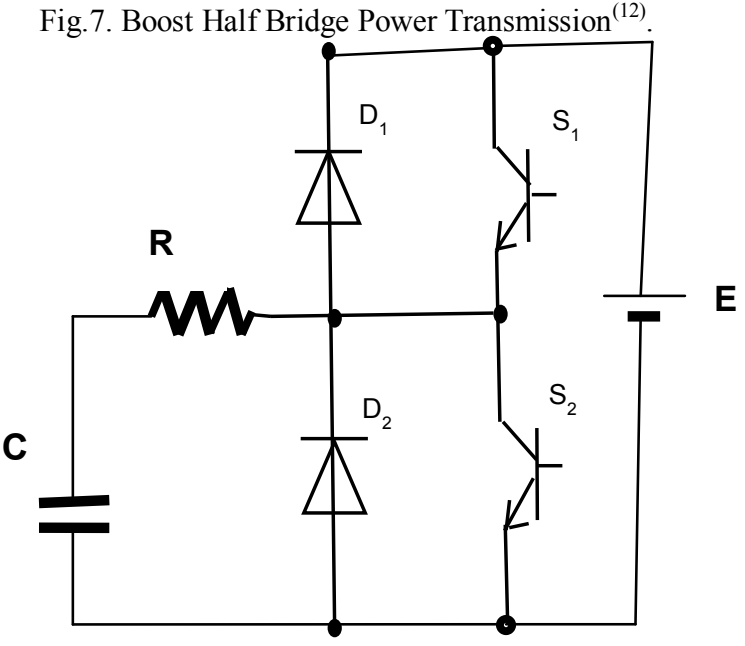

Fig.8. Circuit Configuration of Buck Inverter.

\section{Comparison of Estimated Transmission Efficiency}

\subsection{Local transmission efficiency}

The number of component of circuit construction gives an influence for efficiency. The estimated efficiency will be calculated by means of number of stages by which 


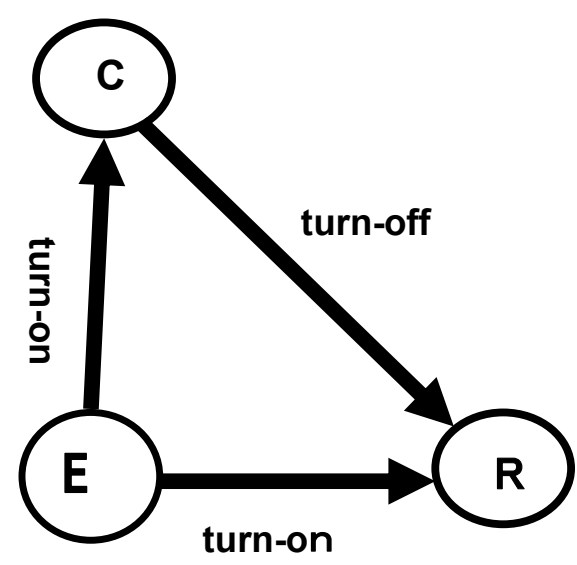

Fig.9. Flowchart of Buck Inverter.

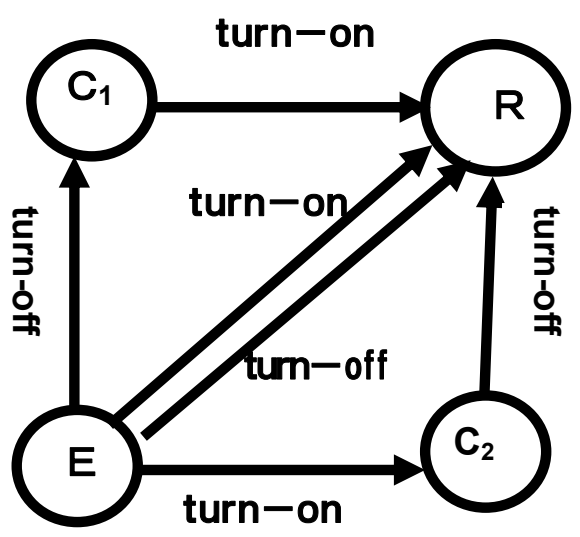

Fig.10. Flowchart of Half Bridge as $\mathrm{S}_{1}$ Operation.

goodness of converter will be estimated. In order to estimate the whole power transmission efficiency, efficiency of one transmission is assumed to be, for example, $90 \%$.

(a) The proposed parallel load (Fig.5) Following each percent shows the transmitted power considering the transmission efficiency. Each transmission power is shown as follows;

$\mathrm{E} \rightarrow \mathrm{R}(90 \%), \mathrm{E} \rightarrow \mathrm{L}(90 \%), \mathrm{L} \rightarrow \mathrm{R}(54 \%) \mathrm{L} \rightarrow \mathrm{C}(36 \%)$, $\mathrm{C} \rightarrow \mathrm{R}(36 \%)$

The quantities of transmission power are represented by weighing method. Total efficiency is $\underline{\underline{84.0 \%}}$

(b) B H B method (Fig. 7)

$\mathrm{E} \rightarrow \mathrm{L}(90 \%), \mathrm{E} \rightarrow \mathrm{C}(90 \%), \mathrm{L} \rightarrow \mathrm{C}(90 \%) \mathrm{C} \rightarrow \mathrm{R}(90 \%)$ 。

The quantities of transmission power are represented by weighing method. Total efficiency is $\underline{\underline{77.0 \%}}$.

The efficiency is about reduced by $10 \%$. For proposed circuit is improved because of direct transmission for half period.

(c) Buck inverter (Fig.8) Weighting transmission efficiency is

$\mathrm{E} \rightarrow \mathrm{R}(90 \%), \mathrm{E} \rightarrow \mathrm{C}(90 \%), \mathrm{C} \rightarrow \mathrm{R}(90 \%)$ 。

The quantities of transmission power are represented by weighing method. Total efficiency is $85.5 \%$.

(d) .Conventional half bridge inverter (Fig. 10
$\mathrm{E} \rightarrow \mathrm{R}(90 \%), \mathrm{E} \rightarrow \mathrm{C}_{1}(90 \%), \mathrm{C}_{1} \rightarrow \mathrm{R}(90 \%)$ 。 In the following switching, operation is transferred to the charging of $\mathrm{C}_{2}$, and the current of $\mathrm{R}$ is switched to the charge of $\mathrm{C}$, and direction of current is also switched as $\mathrm{E} \rightarrow \mathrm{R}(90 \%), \mathrm{E}$ $\rightarrow \mathrm{C}_{2}(90 \%), \mathrm{C}_{2} \rightarrow \mathrm{R}(90 \%)$ 。

The total efficiency is $\underline{\underline{85.5 \%}}$.

\subsection{Comparison of efficiency}

Table 1 Characteristics of Various Converter.

\begin{tabular}{|l|c|c|c|c|}
\hline & Eff \% & Num. & $\begin{array}{l}\text { Load } \\
\text { V }\end{array}$ & Max.curren \\
\hline Buck-Boost Inv & $\mathbf{8 4 . 0}$ & $\mathbf{8}$ & $E$ & $I * 2$ \\
\hline Buck-Half-Bridg & 77.0 & 9 & $E$ & $I * 2$ \\
\hline Buck Inverter & $\mathbf{8 5 . 5}$ & 7 & $E / 2$ & $I$ \\
\hline Half Bridge Inv. & $\mathbf{8 5 . 5}$ & $\mathbf{8}$ & $E / 2$ & $I$ \\
\hline
\end{tabular}

Table 1 represents above mentioned efficiency for comparison. Upper two stages and lower two ones are analogous construction, respectively. Comparing Buck-boost Inverter with B H B, for load voltage, the supply voltage can be applied directly as advantage. The maximum, current of component is double of load current as dis advantage. For proposed converter, the number of component is reduced from nine to eight, which brings efficiency improvement.。 In lower two stages, the load voltage is reduced to half of the supply voltage as dis advantage, but the load current does not flow over load current. Comparing both inverters, the number of component of Buck Inverter is reduced by unity.

\section{Conclusions}

The paper is proposed and presented whose idea is obtained from unified inverter circuit constructed by chopper and inverter circuit. In the first version which can realize sinusoidal wave, the number of the circuit construction can be made minimum. The number of the conventional corresponding circuit configuration is totally ten, while for the proposed construction, the number is eight. This result is the reason why the proposed inverter is called as minimum circuit construction. In parallel load method, which can be developed to dc to dc converter, as the number of conversion stages is reduced satisfactorily, an improved efficiency can be achieved. Finally as looking at the whole view compared with the usual half bridge construction, one of double supply capacitors is replaced by dc power supply from regular position, that is an interesting appearance.

\section{Acknowledgements}

This research is mostly supported by a grant of the NEDO (New Energy and Industry Technology Development Organization). We would like to express our appreciation to who it may concern about this project.

\section{References}

[1] R.O.Caceres and Barbi, "A boost DC-AC converter; analysis, design and experimentation," Trans. On Power Electronics, vol.14, pp.134-141,1999

[2] Minsoo Jang and Vassilios G. Agelidis; "A minimum power processing 
stage fuel-cell energy system based on a boost-inverter with a bidirectional backup battery storage", Trans. On Power Electronics, vol.26, pp.1568-1577, 2011

[3] Minsoo Jang, Takyun Kim, Vassilios G. Agelidis; "Design and Implementation of a $200 \mathrm{kHz}$ Single-Phase Boost-Inverter Using Silicon Carbide Semiconductors",Proceedings of IEEE IECON 2015, Yokohama, pp.2241-2246, 2015-11

[4] P.Sanchis,A.Ursaea,F.Gubia and L.Marroyo;"Boost DC-AC inverter: a new control strategy",Trans. On Power Electronics,vol.20, pp.343353,2005

[5] C.-M. Wang;"A novel single stage full bridge buck-boost inverter", IEEE Trans. on Power Electronics,vol.19, pp.150-159,2004

[6] C.-M. Wang;"A novel single stage series resonant buck-boost inverter", IEEE Trans. on Industrial Electronics,vol.52, pp. 10191108,2005

[7] Keiju Matsui, Eiji Oishi,Yasutaka Kawata, Mikio Yasubayashi, Masayoshi Umeno, Hideo Uchida, Masaru Hasegawa, "Pursuit for Simple Power Conditioner and System Construction of Photovoltaic
Power Generation as Veranda Solar", Proceedings of the 3rd International Conference on Industrial Application Engineering, pp.531-536, 2015-3

[8] Sinichi Jinno, “Chopper Type Inverter Circuit", Patent publication number -2006-174653,(2006-6)

[9] Fang Zheng Peng:"Z-Source Inverter", IEEE Transactions on Industry Applications, Vol. 39, No. 2, pp.504-510,March/April 2003

[10] Keiju Matsui, Eiji Oishi, Yasutaka Kawata, Mikio Yasubayashi, Masayoshi Umeno, Hideo Uchida, Masaru Hasegawa: "Proposal of Boost-Inverter having Voltage Double Capability", The Proceedings of ICEMS-2016, DS1G-2-1, 2016-11

[11] Keiju Matsui, Eiji Oishi, Yasutaka Kawata, Mikio Yasubayashi, Masayoshi Umeno, Hideo Uchida,Masaru Hasegawa: "Pursuit of Simple PCS for Photovoltaic Power Generation - Optimum Waveforms", IEEE-Intelec2015, pp.915-920, 2015.10

[12] Hiroshi Unno, Masanori Hayashi,Yoshiaki Matsuda : "High Efficiency Low Output Voltage DC/DC Power Supply", IEICE, 2000-58, pp.41- 48, 2001-2 